Pacific Journal of Mathematics

UNIFORM APPROXIMATION BY ELEMENTS OF A CONE OF

WALTER ROTH 


\title{
UNIFORM APPROXIMATION BY ELEMENTS OF A CONE OF REAL-VALUED FUNCTIONS
}

\author{
WALTER ROTH
}

The present paper gives conditions for approximation of all functions of a subspace $M$ of $C(K)$, where $K$ is a compact Hausdorff space by elements of an "admissible" subcone $N$ of $M$. This implies generalizations of well-known theorems of Bauer and Stone-Weierstrass.

1. Preliminaries. Let $K$ be a compact Hausdorff space, $M$ a linear space of real-valued continuous functions on $K$. A subcone $N$ of $M$ is said to be admissible if it separates the points of $K$ and contains the constant functions. Denote by $N^{c}$ the set of functions in $C(K)$ that are the pointwise sup of finitely many functions in $N$. Let $<_{N}$ be the relation on $\Omega_{K}$ (the set of probability measures on $K$ ) defined by: $\mu<_{N} \nu$ iff for every $f$ in $N \mu(f) \leqq \nu(f)$. Then $<_{N^{c}}$ is an order relation on $\Omega_{K}$. For every $\mu \in \Omega_{K}$ there exists an $N^{c}$-maximal (maximal with respect to $<_{N^{c}}$ ) measure $\nu \in \Omega_{K}$ such that $\nu \underset{N^{c}}{>} \mu$ (see [1], I.5) The $N^{c}$-maximal measures live on every Baire set containing the Choquet boundary $C h_{K}(N)$ of $N$.

$$
C h_{K}(N)=\left\{x \in K \mid \text { for every } \mu \in \Omega_{K} u>_{N} \epsilon_{x} \text { implies } \mu=\epsilon_{x}\right\} \text { ( } \epsilon_{x} \text { is }
$$
the point measure at $x$ ). For $f \in C(K)$ the upper and lower envelopes are defined by

$$
\bar{f}_{N}=\inf \{h \in-N \mid h>f\} \quad f_{N}=\sup \{h \in N \mid h<f\}
$$

$\bar{f}_{N}$ resp. $f_{N}$ are upper resp. lower-semi-continuous and coincide with $f$ on $C h_{K}(N)$. For every $N^{c}$-maximal measure $\mu \in \Omega_{K}$ we have $\mu(f)=$ $\mu\left(\bar{f}_{N}\right)=\mu\left(f_{N}\right)$.

\section{An approximation-theorem: conditions for} measures.

THEOREM 1. Let $M$ be a linear space of continuous real-valued functions on the compact Hausdorff space $K$ and $N$ an admissible cone. $N$ is uniformly dense in $M$ (dense with respect to the sup-norm) if and only if 
(1) For all $N^{c}$-maximal measures $\mu, \nu$ in $\Omega_{K} \mu>_{N} \nu$ implies $\mu>_{M} \nu$, and one of the following conditions holds

(2a) Every $M^{c}$-maximal measure of $\Omega_{K}$ is $N^{c}$-maximal.

(2b) $f_{N}=f$ for every $f$ in $M$.

If $K$ is metrizable or if $C h_{K}(N)$ is compact (2a) and (2b) can be replaced by $(2 \mathrm{c}) C h_{K}(N)=C h_{K}(M)$

The proof will use

Lemma 1. N is uniformly dense in $M$ if and only if for all $\mu, \nu$ in $\Omega_{K} \mu \underset{N}{>} \nu$ implies $\mu \underset{M}{>} \nu$.

This Lemma is an immediate consequence of the Hahn-Banach Theorem and still holds if both $N$ and $M$ are admissible cones. Using the fact that the uniform closure of $N$ in $C(K)$ is given by

$\bar{N}=\{f \in C(K) \mid$ such that for every measure $\lambda$ on $K \lambda(N) \geqq 0$ implies $\lambda(f) \geqq 0$.

we decompose each such measure $\lambda=\frac{1}{2}\|\lambda\|(\mu-\nu)$, where $\mu, \nu \in \Omega_{K}$ and deduce Lemma 1.

To prove the theorem

( $\alpha$ ) suppose (1) and (2a) hold, $\mu, \nu \in \Omega_{K}$ such that $\mu>_{N} \nu$.

Let $\mu^{\prime}$ be $M^{c}$-maximal and $\mu^{\prime}{\overrightarrow{M^{c}}}^{c} \mu \nu^{\prime}$ be $M^{c}$-maximal and $v^{\prime}{\overrightarrow{M^{c}}}^{c} \nu$. Then $\mu^{\prime}$ and $\nu^{\prime}$ are $N^{c}$-maximal (2a) and $\mu^{\prime} \underset{N^{c}}{>} \mu, \nu^{\prime} \underset{N^{c}}{>} \nu$ because $\left.N^{c} \subset M^{c}\right) . \quad M$ is a linear space, therefore $<_{M}$ implies $>_{M}$ (indeed $<_{M}$ is an equivalence relation $\widetilde{M}$ ) and we conclude

$$
\begin{aligned}
& \mu^{\prime} \widetilde{M} \mu \\
& \nu^{\prime} \widetilde{M} \nu
\end{aligned} \Rightarrow \mu^{\prime}(f)=\mu(f) \geqq \nu(f)=\nu^{\prime}(f) \text { for every } f \text { in } N .
$$

i.e. $\mu^{\prime}>_{N} \nu^{\prime}$ which implies by (1) $\mu^{\prime}>_{M} \nu^{\prime}$, hence $\mu \underset{M}{>_{M}} \nu$. From Lemma 1 we deduce $M \subset \bar{N}$. 
( $\beta$ ) (2b) implies (2a) because for every $f \in M$ the set $\left\{h \in N^{c} \mid h<f\right\}$ is directed upward converging pointwise (2b) to the continuous function $f$. By Dini's Lemma the convergence is uniform, hence $f \in \overline{N^{c}}, M \subset \overline{N^{c}}, M^{c} \subset \overline{N^{c}} . \quad N^{c}$ and $M^{c}$ define the same order relation on $\Omega_{K}$.

( $\gamma$ ) Suppose $K$ is metrizable or $C h_{K}(N)$ is compact. In this case $C h_{K}(N)=C h_{K}(M)$ implies (2a) because every measure in $\Omega_{K}$ is $N^{c_{-}}$ maximal (resp. $M^{c}$ - maximal) then if and only if it lives on $C h_{K}(N)$ (resp. $\left.C h_{K}(M)\right)$.

As a corollary of Theorem 1 we find a well-known theorem ([1], Th. II.4.5) on affine functions on a compact convex set:

Corollary 1. Let $A$ be the set of the affine continuous functions on the compact convex set $X, E x(X)$ the extreme points of $X$, $f: \operatorname{Ex}(X) \rightarrow \mathbf{R}$ a bounded continuous function (in the relative topology on $\operatorname{Ex}(X))$.

$f$ can be extended to a function in $A$ if and only if

$$
\begin{aligned}
& \text { (1) } \bar{f}=f \text { on } \overline{\operatorname{Ex}(X)} \quad(\bar{f}=\inf \{g \in A \mid g>f \quad \text { on } E x(X)\} \\
& f=\sup \{g \in A \mid g<f \text { on } \operatorname{Ex}(X)\})
\end{aligned}
$$

and

(2) For all $A^{c}$-maximal measures $\mu, \nu$ in $\Omega_{x} \mu>_{A} \nu$ implies $\mu(\bar{f})=$ $\nu(\bar{f})$.

To prove the corollary suppose $K=\overline{\operatorname{Ex(X)}}, N=\left.A\right|_{K}$ and $M=$ $N+\mathbf{R} \cdot f$, where $f=\left.f\right|_{K}=\left.\bar{f}\right|_{K} \in C(K)$. Clearly $N$ is closed in $C(K)$ and from Theorem $1(2 \mathrm{~b})$ we see that $N$ is uniformly dense in $M$, i.e. $N=M$.

REMARK. It is easy to show that if $N$ is a linear space too, then condition (1) in Theorem 1 can be reduced to the comparison of maximal simplex measure (c.f. [1], Chapter I.6), i.e.

(1') For all $N^{c}$-maximal simplex measures $\mu, \nu$ in $\Omega_{K} \mu>_{N} \nu$ implies $\mu>_{M} \nu$.

3. Conditions for the order relation. In this section we are going to replace (1) in Theorem 1 by conditions in terms of the order relation $\leqq$ in the function sets $N$ and $M$. We use a simple generalization of a theorem of Cartier, Fell and Meyer: 
LEMMA 2. Let $M$ be a linear space of continuous real-valued functions on the compact Hausdorff space $K, N$ an admissible cone in $M$. Suppose that for every function $f \in M$ the set

$$
\{g \in N \mid g<f\}
$$

is directed upward. Then for all $\mu, \nu, \nu_{1}, \nu_{2} \in \Omega_{k}, \lambda_{1}, \lambda_{2} \geqq 0$ such that $\mu \underset{N}{>} \nu, \lambda_{1}+\lambda_{2}=1$ and

$$
\text { for every } f \text { in } M \nu(f)=\lambda_{1} \nu_{1}(f)+\lambda_{2} \nu_{2}(f)
$$

there exist $\mu_{1}, \mu_{2} \in \Omega_{K}$ such that $\mu_{1}>\nu_{1}, \mu_{2}>\nu_{2}$ and

$$
\text { for every fin } M \mu(f)=\lambda_{1} \mu_{1}(f)+\lambda_{2} \mu_{2}(f) \text {. }
$$

To prove the lemma assume $\lambda_{1}, \lambda_{2}>0$. Define $\phi: M \times M \rightarrow \mathbf{R}$ by

$$
\phi(f, g)=\lambda_{1} \nu_{1}\left(\bar{f}_{N}\right)+\lambda_{2} \nu_{2}\left(\bar{g}_{N}\right)
$$

Then

$(\alpha) \phi$ is positive subhomogeneous.

( $\beta$ ) For every $f \in M$, also $-f \in M$ and therefore

$\{h \in N \mid h<-f\}$ is directed upward, hence

$\{h \in-N \mid h>f\}$ is directed downward, hence

$\mu\left(\bar{f}_{N}\right)=\inf \{\mu(h) \mid h \in-M, h>f\}$

$\leqq \inf \{\nu(h) \mid h \in-N, h>f\}=\nu\left(\bar{f}_{N}\right)$.

$(\gamma)$ Suppose $F=\{(f, f) \in M \times M\}$ and

$$
\psi_{0}: F \rightarrow R, \quad \psi_{0}(f, f)=\mu(f) .
$$

Then $\psi_{0}(f, f)=\mu(f) \leqq \mu\left(\bar{f}_{N}\right) \leqq \nu\left(\bar{f}_{N}\right)=\phi(f, f)$.

( $\delta$ ) By the Hahn-Banach Theorem there exists a linear extension

$$
\psi: M \times M \rightarrow \mathbf{R} \text { of } \psi_{0} \text { such that } \psi \leqq \phi .
$$

( $\epsilon)$ Let $\mu_{1}, \mu_{2}$ be probability measures on $K$ such that for every $f$ in $M$

$$
\mu_{1}(f)=1 / \lambda_{1} \psi(f, 0) \quad \text { and } \quad \mu_{2}(f)=1 / \lambda_{2} \psi(0, f)
$$


Then we conclude

For every $f$ in $M \mu(f)=\psi_{0}(f, f)=\psi(f, f)=\lambda_{1} \mu_{1}(f)+\lambda_{2} \mu_{2}(f)$.

For every $f$ in $-N \mu_{1}(f)=1 / \lambda_{1} \psi(f, 0) \leqq 1 / \lambda_{1} \phi(f, 0)=\nu_{1}\left(\bar{f}_{N}\right)=\nu_{1}(f)$.

Hence $\nu_{1}<_{N} \mu_{1}$ and $\nu_{2}<_{N} \mu_{2}$

which proves the lemma. Now we can state:

THEOREM 2. Let $M$ be a linear space of continuous real-valued functions on the compact Hausdorff space $K, N$ an admissible cone in $M$. $N$ is uniformly dense in $M$ if and only if

(1) For every $f \in M$ the set

$$
\{g \in N \mid g<f\}
$$

is directed upward and

(2) $C h_{K}(N)=C h_{K}(M)$.

Proof. Of course $M \subset \bar{N}$ implies (1) and (2). Suppose now (1) and (2) hold and

$$
\begin{aligned}
& R=\left\{(\nu, \mu) \in M^{\prime} \times M^{\prime} \mid \quad \mu, \nu \geqq 0, \quad\|\mu\|=\|\nu\|=1, \quad \nu<_{N} \mu\right\} \\
& S=\left\{(\mu, \mu) \in M^{\prime} \times M^{\prime} \mid \quad \mu \geqq 0 \quad\|\mu\|=1\right\}
\end{aligned}
$$

Both $R$ and $S$ are compact convex in the weak topology on $M^{\prime} \times M^{\prime}$, $S \subset R$. We show $S=R$ and apply Lemma 1 .

Lemma 2 shows that

$$
\operatorname{Ex}(R) \subset\left\{\left(\epsilon_{x}, \mu\right) \in R \mid x \in C h_{K}(M)\right\}
$$

because every $\mu \in M^{\prime}, \mu \neq \epsilon_{x}$ for every $x \in C h_{K}(M)$, is a convex combination of two different elements of $M^{\prime}$. From $C h_{K}(N)=$ $C h_{K}(M)$ we conclude now

$$
\left(\epsilon_{x}, \mu\right) \in \operatorname{Ex}(R) \Rightarrow \epsilon_{x}<_{N} \mu \Rightarrow \epsilon_{x}=\mu \Rightarrow\left(\epsilon_{x}, \mu\right) \in S
$$

hence $\operatorname{Ex}(R) \subset S$, which implies $R=S$ and proves the theorem.

4. Applications: The case $M=C(X)$. If we choose $M=C(X)$ Theorem 2 leads to generalizations of theorems of Bauer and of Stone-Weierstrass. 
THEOREM 3. Let $N$ be an admissible cone of continuous realvalued functions on the compact Hausdorff space $K$. The following conditions are equivalent:

(1) $N$ is uniformly dense in $C K$ ).

(2) $\bar{N}$ is maximum-stable, i.e. $N^{c} \subset \bar{N}$, and $C h_{K}(N)=$ $C h_{K}(N-N)$.

(3) For all $f, g \in N$ inf $\{h \in N \mid h>f \vee g\} \in \bar{N}$ ( $f \vee g$ is the pointwise sup of $f$ and $g$ )

$$
\text { and } C h_{K}(N)=C h_{K}(N-N) \text { and } \overline{C h_{K}(-N)}=K \text {. }
$$

Proof. The implications (1) $\Rightarrow$ (2) and (1) $\Rightarrow$ (3) are trivial. Suppose (2) holds: Theorem 2 shows that $N-N \subset \bar{N}$, hence $\bar{N}$ is a linear space. $\quad N^{c} \subset \bar{N}$ therefore implies $\left(N^{c}-N^{c}\right) \subset \bar{N}$. From the classical Stone-Weierstrass theorem we know that $N^{c}-N^{c}$ is dense in $C(K)$ which proves $\bar{N}=C(K)$.

Suppose now (3) holds. We show that $N^{c} \subset \bar{N}$ and therefore (3) implies (2): Let $f, g \in N$. Then

$$
h={\overline{f \vee g_{(-N)}}}_{(\inf }\{j \in N \mid j>f \vee g\} \in \bar{N}
$$

Now $\left.h\right|_{C h_{K}(-N)}=\left.f \vee g\right|_{C h_{K}(-N)}$ and $\overline{C h_{K}(-N)}=K$ imply $h=f \vee g \in \bar{N}$, hence $N^{c} \subset \bar{N}$.

Bauer's theorem (see [1], Th. II.4.1) is a special case of the following.

TheOREM 4. Let $N$ be a uniformly complete admissible cone of continuous real-valued functions on the compact Hausdorff space $X$, $K=\overline{C h_{X}(N-N)}$. The following conditions are equivalent:

(1) $\left.N\right|_{K}=C(K)$.

(2) For all $N^{c}$-maximal measures $\mu, \nu$ in $\Omega_{x} \mu>_{N} \nu$ implies $\mu=\nu$ and $C h_{X}(N)=K$.

(3) For all $f, g \in N$ inf $\{h \in N \mid h>f \vee g\} \in N$ and $C h_{X}(N)=$ $C h_{X}(N-N)$ and $C h_{X}(-N)=K$.

Proof. Clearly $\left.N\right|_{K}$ is closed in $C(K)$. (1) $\Leftrightarrow(2)$ is an immediate consequence of Theorem 1. (1) $\Rightarrow(3)$ is trivial. To prove the implication (3) $\Rightarrow$ (1) we take $f, g \in N$. (3) guarantees the existence of an $h \in N$ such that $h=\inf \{j \in N \mid j>f \vee g\}$. From $\overline{C h_{K}(-N)}=K$ we conclude now $\left.h\right|_{K}=\left.f \vee g\right|_{K}$. Hence $\left.N\right|_{K}$ is maximum stable and Theorem (3) proves $\left.N\right|_{K}=C(K)$. (Obviously $C h_{K}\left(\left.N\right|_{K}\right)=C h_{X}(N)$.) 


\section{Examples.}

1. Suppose $K$ is the finite set $\left\{x_{1}, \cdots, x_{n}\right\}, N$ an admissible cone of functions (here real-valued finite sequences) on $K$. Then $C h_{K}(N)$ consists of all $x_{j} \in K$ such that there exists $f \in N$, such that $f\left(x_{i}\right)<f\left(x_{j}\right)$ for all $i \neq j$. (see [4], §7, Ex. 3). In this case Theorem 3 states:

$N=C(K)=\mathbf{R}^{n}$ iff for all $f, g \in N$ inf $\{h \in N \mid h>f \vee g\} \in N$ and for every $x_{j} \in K$ there are $f, g \in N$ such that if $i \neq j f\left(x_{i}\right)<f\left(x_{j}\right)$ and $g\left(x_{i}\right)>g\left(x_{j}\right)$.

2. Let $K$ be the compact subset $\left\{1, \frac{1}{2}, \frac{1}{3}, \frac{1}{4}, \cdots, 0\right\}$ of $\mathbf{R}$. Then $C(K)$ is the set of all convergent real-valued sequences.

$$
N=\left\{(y)_{n} \in C(K) \mid(y)_{n}=\left(\alpha_{1}, \alpha_{2}, \cdots \alpha_{k}\right)+\lambda\left(1, \frac{1}{2}, \frac{1}{3}, \frac{1}{4}, \cdots, 0\right)+r,\right.
$$

where $\left.\quad r \in \mathbf{R}, k \in \mathbf{N}, \lambda \leqq \min \left\{0, \alpha_{1}, \alpha_{2}, \cdots \alpha_{k}\right\}\right\}$

defines an admissible cone in $C(K)$ with the properties:

(1) $N \cap(-N)=\mathbf{R}$ :

Let $f \in N \cap(-N)$. Then

$$
\begin{aligned}
& f=\left(\alpha_{1}, \cdots \alpha_{k}\right)+\lambda\left(1, \frac{1}{2}, \cdots\right)+r=-\left(\beta_{1}, \cdots \beta_{k}\right)-\rho\left(1, \frac{1}{2}, \cdots\right)=r^{\prime} \\
& \Rightarrow \lambda=-\rho=0 \quad \text { because } \quad \lambda, \rho \leqq 0 \\
& \Rightarrow \alpha_{1}, \cdots, \alpha_{k} \geqq 0, \quad \beta_{1}, \cdots, \beta_{k} \geqq 0 . \\
& \Rightarrow \alpha_{1}=-\beta_{1}=0, \cdots \quad \alpha_{k}=-\beta_{k}=0 . \\
& \Rightarrow f \in \mathbf{R} .
\end{aligned}
$$

(2) Every $x \in K$ is a maximum point of an element of $N$. (For $x=0$ choose $\left.\alpha_{i}=0, \lambda=-1\right)$. Therefore $C h_{K}(N)=K$ and $C h_{K}(N)=C h_{K}(N-N)=K$.

Every $1 / n \in K$ is a minimum point of an element of $N$, hence $C h_{K}(-N)=K$.

(3) $N$ is maximum-stable.

From Theorem 3 we conclude that $N$ is uniformly dense in $C(X)$.

\section{REFERENCES}

1. E. M. Alfsen, Compact Convex Sets and Boundary Integrals, Springer, Berlin 1971.

2. H. Bauer, Harmonische Räume und ihre Potentialtheorie, Lecture Notes 22, Springer 1966.

3. Silovscher Rand und Dirichletsches Problem, Ann. Inst. Fourier, 11 (1961), 89-136.

4. N. Bourbaki, Éléments de Mathématique, Intégration. Livre VI.

5. Robert R. Phelps, Lectures on Choquet's Theorem, D. van Nostrand Comp. Inc., Princeton 1966.

6. W. Roth, Approximation und Fortsetzung stetiger Funktionen auf kompakten Mengen, Darmstadt, 1973.

Received January 17, 1974.

University of CALIFornia, Berkeley 



\section{PACIFIC JOURNAL OF MATHEMATICS}

\section{EDITORS}

RICHARD ARENS (Managing Editor)

University of California

Los Angeles, California 90024

\author{
R. A. Beaumont \\ University of Washington \\ Seattle, Washington 98105
}

\section{J. DugundII}

Department of Mathematics

University of Southern California

Los Angeles, California 90007

D. Gilbarg and J. Milgram

Stanford University

Stanford, California 94305

\section{ASSOCIATE EDITORS}
E. F. BECKENBACH
B. H. NeumanN
F. WoLF
K. YoshidA

\section{SUPPORTING INSTITUTIONS}

\author{
UNIVERSITY OF BRITISH COLUMBIA \\ CALIFORNIA INSTITUTE OF TECHNOLOGY \\ UNIVERSITY OF CALIFORNIA \\ MONTANA STATE UNIVERSITY \\ UNIVERSITY OF NEVADA \\ NEW MEXICO STATE UNIVERSITY \\ OREGON STATE UNIVERSITY \\ UNIVERSITY OF OREGON \\ OSAKA UNIVERSITY
}

\author{
UNIVERSITY OF SOUTHERN CALIFORNIA \\ STANFORD UNIVERSITY \\ UNIVERSITY OF TOKYO \\ UNIVERSITY OF UTAH \\ WASHINGTON STATE UNIVERSITY \\ UNIVERSITY OF WASHINGTON \\ AMERICAN MATHEMATICAL SOCIETY
}

The Supporting Institutions listed above contribute to the cost of publication of this Journal, but they are not owners or publishers and have no responsibility for its contents or policies.

Mathematical papers intended for publication in the Pacific Journal of Mathematics should be in typed form or offset-reproduced (not dittoed), double spaced with large margins. Underline Greek letters in red, German in green, and script in blue. The first paragraph or two must be capable of being used separately as a synopsis of the entire paper. Items of the bibliography should not be cited there unless absolutely necessary, in which case they must be identified by author and Journal, rather than by item number. Manuscripts, in duplicate, may be sent to any one of the four editors. Please classify according to the scheme of Math. Reviews, Index to Vol. 39. All other communications should be addressed to the managing editor, or Elaine Barth, University of California, Los Angeles, California, 90024.

100 reprints are provided free for each article, only if page charges have been substantially paid. Additional copies may be obtained at cost in multiples of 50.

The Pacific Journal of Mathematics is issued monthly as of January 1966. Regular subscription rate: $\$ 72.00$ a year (6 Vols., 12 issues). Special rate: $\$ 36.00$ a year to individual members of supporting institutions.

Subscriptions, orders for back numbers, and changes of address should be sent to Pacific Journal of Mathematics, 103 Highland Boulevard, Berkeley, California, 94708.

PUBLISHED BY PACIFIC JOURNAL OF MATHEMATICS, A NON-PROFIT CORPORATION Printed at Jerusalem Academic Press, POB 2390, Jerusalem, Israel.

\section{Copyright (C) 1975 Pacific Journal of Mathematics All Rights Reserved}




\section{Pacific Journal of Mathematics

Vol. 60, No. $2 \quad$ October, 1975

Waleed A. Al-Salam and A. Verma, A fractional Leibniz q-formula ........... 1

Robert A. Bekes, Algebraically irreducible representations of $L_{1}(G) \ldots \ldots \ldots \ldots 11$

Thomas Theodore Bowman, Construction functors for topological

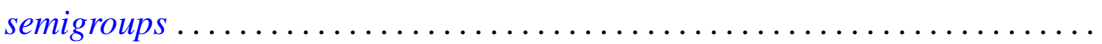

Stephen LaVern Campbell, Operator-valued inner functions analytic on the

closed disc. II .........................................

Leonard Eliezer Dor and Edward Wilfred Odell, Jr., Monotone bases in $L_{p} \ldots \ldots$.

Yukiyoshi Ebihara, Mitsuhiro Nakao and Tokumori Nanbu, On the existence of

global classical solution of initial-boundary value problem for

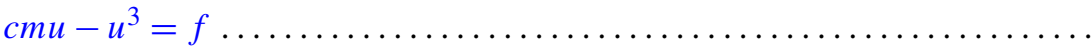

Y. Gordon, Unconditional Schauder decompositions of normed ideals of

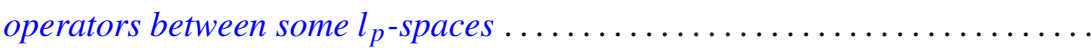

Gary Grefsrud, Oscillatory properties of solutions of certain nth order functional

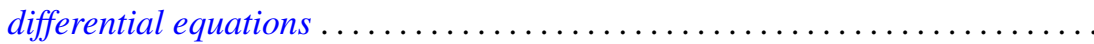

Irvin Roy Hentzel, Generalized right alternative rings ...................

Zensiro Goseki and Thomas Benny Rushing, Embeddings of shape classes of compacta in the trivial range .................................

Emil Grosswald, Brownian motion and sets of multiplicity . .

Donald LaTorre, A construction of the idempotent-separating congruences on a

bisimple orthodox semigroup .

Pjek-Hwee Lee, On subrings of rings with involution ...

Marvin David Marcus and H. Minc, On two theorems of Frobenius ...

Michael Douglas Miller, On the lattice of normal subgroups of a direct

product. .

Grattan Patrick Murphy, A metric basis characterization of Euclidean space

Roy Martin Rakestraw, A representation theorem for real convex functions ....

Louis Jackson Ratliff, Jr., On Rees localities and $H_{i}$-local rings ...

Simeon Reich, Fixed point iterations of nonexpansive mapping . .

Domenico Rosa, $B$-complete and $B_{r}$-complete topological algebras ...

Walter Roth, Uniform approximation by elements of a cone of real-valued

functions ....

Helmut R. Salzmann, Homogene kompakte projektive Ebenen

Jerrold Norman Siegel, On a space between $B H$ and $B_{\infty} \ldots$

235

Robert C. Sine, On local uniform mean convergence for Markov operators

James D. Stafney, Set approximation by lemniscates and the spectrum of an

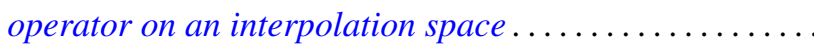

Árpád Száz, Convolution multipliers and distributions .......

Kalathoor Varadarajan, Span and stably trivial bundles ..........

Robert Breckenridge Warfield, Jr., Countably generated modules over

commutative Artinian rings....................... 